\title{
An ISO-SWS survey of molecular hydrogen in starburst and Seyfert galaxies ${ }^{\star}$
}

\author{
D. Rigopoulou ${ }^{1}$, D. Kunze ${ }^{1}$, D. Lutz ${ }^{1}$, R. Genzel ${ }^{1}$, and A. F. M. Moorwood ${ }^{2}$ \\ 1 Max-Planck-Institut für extraterrestrische Physik, Postfach 1312, 85741 Garching bei München, Germany \\ 2 European Southern Observatory, Karl-Schwarzschild-Str. 2, 85748 Garching bei München, Germany
}

Received 12 November 2001 / Accepted 15 April 2002

\begin{abstract}
We present results from a survey of molecular hydrogen emission from a sample of Starburst and Seyfert galaxies carried out with the Infrared Space Observatory (ISO). Pure rotational $\mathrm{H}_{2}$ emission has been detected in a number of extragalactic objects and a variety of environments. A number of transitions from $S(7)$ to $S(0)$ are detected in both starbursts and Seyferts. Using excitation diagrams we derive temperatures and masses of the "warm" molecular hydrogen. We find that the temperature of the "warm" gas is similar in starbursts and Seyferts (those Seyferts for which we have firm detections of the $\mathrm{S}(0)$ line) with a value of around $T \sim 150 \mathrm{~K}$. This "warm" gas accounts for as much as $10 \%$ of the total galactic mass (as probed by CO molecular observations) in starbursts. The fraction of "warm" gas is overall higher in Seyferts, ranging between $2-35 \%$. We then investigate the origin of the warm $\mathrm{H}_{2}$ emission. Comparison with published theoretical models and Galactic templates implies that although emission from photodissociation regions (PDR) alone could explain the emission from starbursts and Seyferts, most likely a combination of PDR, shock emission and gas heated by X-rays (mostly for the Seyferts) is responsible for $\mathrm{H}_{2}$ excitation in extragalactic environments. Finally, we find that although $\mathrm{PAH}$ and $\mathrm{H}_{2}$ line emission correlate well in starbursts and the large scale emission in AGN, $\mathrm{H}_{2}$ emission is much stronger compared to PAH emission in cases where a "pure" AGN dominates the energy output.
\end{abstract}

Key words. galaxies: active - galaxies: starburst - infrared: galaxies

\section{Introduction}

Using the Short Wavelength Spectrometer (SWS; De Graauw et al. 1996) on board the Infrared Space Observatory (ISO; Kessler et al. 1996), we have performed a survey of molecular hydrogen emission from active galaxies displaying a wide range in nuclear activity including pure bona-fide starbursts, Seyfert 2s (some of them with starburst components) and pure Seyfert 1 galaxies. Prior to the ISO mission, extragalactic $\mathrm{H}_{2}$ emission had only been detected in the ro-vibrational lines around $2.1 \mu \mathrm{m}$. Indeed, $\mathrm{H}_{2}$ ro-vibrational emission has been detected in galactic sources (e.g. Usuda et al. 1996), Starburst galaxies (e.g. Joseph et al. 1984), Seyferts (e.g. Moorwood \& Oliva 1988; Fischer et al. 1987) and bright spirals (e.g. Puxley et al. 1988).

Send offprint requests to: D. Rigopoulou, e-mail: dar@mpe.mpg.de

* Based on observations with ISO, an ESA project with instruments funded by ESA Member States (especially the PI countries: France, Germany, The Netherlands and the United Kingdom) and with the participation of ISAS and NASA.
The ro-vibrational lines typically trace $\mathrm{H}_{2}$ gas of masses around $\sim 10^{4-5} M_{\odot}$ and temperatures $\sim 2000 \mathrm{~K}$. This gas can be excited either by collisions (thermal) or by absorption of ultraviolet (UV) photons in the Lyman and Werner electronic bands (912-1108 $\AA$ ), followed by a de-excitation cascade to the ground state (fluorescence). However, gas at these temperatures is a very small fraction (as small as $10^{-6}$ ) of the total amount of $\mathrm{H}_{2}$ gas (e.g. Van der Werf et al. 1993). Since the ro-vibrational lines tend to get faint at lower temperatures, most of our knowledge about the $\mathrm{H}_{2}$ content of galaxies comes from $\mathrm{CO}$ observations assuming a $\mathrm{CO} / \mathrm{H}_{2}$ conversion factor derived from galactic molecular cloud observations. ISO gave the unique opportunity to observe intermediate temperature gas, ie "warm" $\mathrm{H}_{2}$, directly in pure rotational lines. Since transitions with $\Delta J= \pm 1$ are strictly forbidden for the $\mathrm{H}_{2}$ molecule, the rotational ladder consists only of an ortho $(J$ odd) and a para $(J$ even) series of quadrupole transitions.

ISO, and in particular SWS, offered the unique opportunity to detect pure rotational $\mathrm{H}_{2}$ emission in a number of galactic and extragalactic sources, thus studying 
the amount of moderately warm gas in these sources. The spectral range of SWS provides full coverage of a number of transitions (for most galaxies we have observed from the $\mathrm{S}(0)$ to $\mathrm{S}(7)$ transitions) while its spectral resolution is well matched to the typical velocity dispersions of galaxies. Among the first detections of pure rotational $\mathrm{H}_{2}$ emission in galaxies were the detections in NGC 3256 (Rigopoulou et al. 1996), NGC 6946 (Valentijn et al. 1996), NGC 891 (Valentijn \& Van der Werf 1999). However, no study of pure rotational $\mathrm{H}_{2}$ emission for a large number of galaxies has so far appeared.

Here, we present an inventory of $\mathrm{H}_{2}$ emission lines from a number of Starburst and AGN. Our survey includes 12 and 9 Starburst ${ }^{1}$ and Seyfert galaxies, respectively. Temperatures of the warm molecular gas are deduced from excitation diagrams whereas the masses of the warm molecular gas are compared to the total gas content of the galaxies as estimated from molecular CO observations. The $\mathrm{H}_{2}$ excitation mechanism is investigated next. The observations are compared to the predictions of published models both for PDR, shocked emission and X-ray irradiated gas, as well as to Galactic templates. Finally we examine possible correlations between $\mathrm{PAH}$ and $\mathrm{H}_{2}$ emission.

\section{Observations and data reduction}

The data presented here are part of the MPE ISO Guaranteed time project "Bright Galactic Nuclei". The observations were carried out throughout the ISO mission and were all taken in the standard AOT SWS02 mode, i.e. grating line profile scan. The SWS has three different apertures with dimensions (projected on the sky) ap1: $14^{\prime \prime} \times 20^{\prime \prime}$, ap2: $14^{\prime \prime} \times 27^{\prime \prime}$ and ap3: $14^{\prime \prime} \times 20^{\prime \prime}$. The following molecular transitions (and the corresponding apertures) were observed although not in all sources: $(1-0) \mathrm{Q}(3)$ (ap1), (0-0)S(0) (ap3), (0-0)S(1) (ap2), (0-0)S(2) (ap1), (0-0)S(3) (ap3), (0-0)S(5) (ap2), and (0-0)S(7) (ap2). The sample targets are presented in Tables 1 and 2 for the starbursts and Seyferts, respectively. Galaxy names, distances (assuming $H_{0}=75 \mathrm{~km} \mathrm{~s}^{-1} \mathrm{Mpc}^{-1}$ ), extinction towards the ionized medium, and comments are also listed. We note that the SWS aperture sizes are most likely larger than the presumed sizes of the circumnuclear regions for a large fraction of the present samples of Starburst and Seyfert galaxies.

The data were reduced using the Interactive Analysis (IA) Package of the SWS team including sophisticated tools to improve cosmic ray hit removal, dark subtraction, defringing and flat-fielding. The flux calibration is accurate to about $25 \%$ (Schaeidt 1996). The calibration files of June 2000 were used.

\footnotetext{
1 The two components of NGC 3690 have been observed separately NGC 3690A and NGC 3690BC. In the analysis we treat them as two separate systems.
}

Figures 1 and 2 show the observed $\mathrm{H}_{2}$ molecular lines in starburst and Seyfert galaxies ${ }^{2}$. In almost all cases the $\mathrm{S}(1)$ and $\mathrm{S}(5)$ lines are clearly detected in both the Seyfert and the Starburst samples. Tables 3 and 4 give the actual line flux values for starbursts and Seyferts, respectively.

From the sample of 13 starbursts (the two components in NGC 3690 have been observed separately) the $\mathrm{S}(0)$ line was observed in 10 of them and detected in 5 . The $\mathrm{S}(0)$ line was observed in all (9) Seyferts and detected in 4 of them.

For those sources where lines were not detected we quote "good confidence" upper limits. As mentioned earlier a large number of atomic and ionic lines were observed for each source. For the purpose of the current paper we focus on the $\mathrm{H}_{2}$ emission. More detailed analysis for some of the sources can be found in other papers (NGC 4038/39: Kunze et al. 1996; NGC 3256: Rigopoulou et al. 1996; NGC 4151: Alexander et al. 1998; Sturm et al. 1998; Circinus: Moorwood et al. 1996; NGC 1275: Krabbe et al. 2000; NGC 1068: Lutz et al. 2000).

\section{Warm molecular hydrogen emission: Excitation temperatures and masses}

Using the pure rotational $\mathrm{H}_{2}$ lines we can probe the physical conditions of the warm molecular hydrogen in our sample of Starburst and Seyfert galaxies. As we discussed in Sect. 1, the pure rotational lines originate from the warm $(T<2000 \mathrm{~K})$ gas. In what follows we derive excitation temperatures for the "warm" molecular hydrogen gas as well as masses and compare them to the total masses of the galaxies, as probed by observations of the various transitions of the CO molecule. We also compare and discuss the global properties of the "warm" gas in starbursts and Seyferts.

\subsection{Excitation temperatures and masses}

The excitation diagrams in Figs. 3 and 4 show a plot of the natural logarithm of the column density divided by the statistical weight in the upper level of each transition against the energy level. The column density follows from the Boltzmann equation,

$\frac{N_{i}}{N}=\frac{g(i)}{Z\left(T_{\mathrm{ex}}\right)} \times \exp \left(-\frac{T_{i}}{T_{\mathrm{ex}}}\right)$

where $N_{i}$ is the column density of $\mathrm{H}_{2}$ in the $i$ th state, $g(i)$ and $T_{i}$ are the statistical weight and energy level of that state, $N$ is the total column density of $\mathrm{H}_{2}, T_{\text {ex }}$ is the excitation temperature, and $Z\left(T_{\mathrm{ex}}\right)$ is the partition function at $T_{\mathrm{ex}} \cdot N_{i}$ is calculated from the observed quantities according to:

$N_{i}=\frac{\text { flux }(i)}{A(i) \times h \times \nu(i)} \times \frac{4 \pi}{\text { beam }}$

${ }^{2}$ Previously published $\mathrm{H}_{2}$ molecular lines are not shown here. The spectra can be found in: Rigopoulou et al. (1996) for NGC 3256, Kunze et al. (1996) for NGC 4038/39, Spoon et al. (2000) for NGC 4945. 

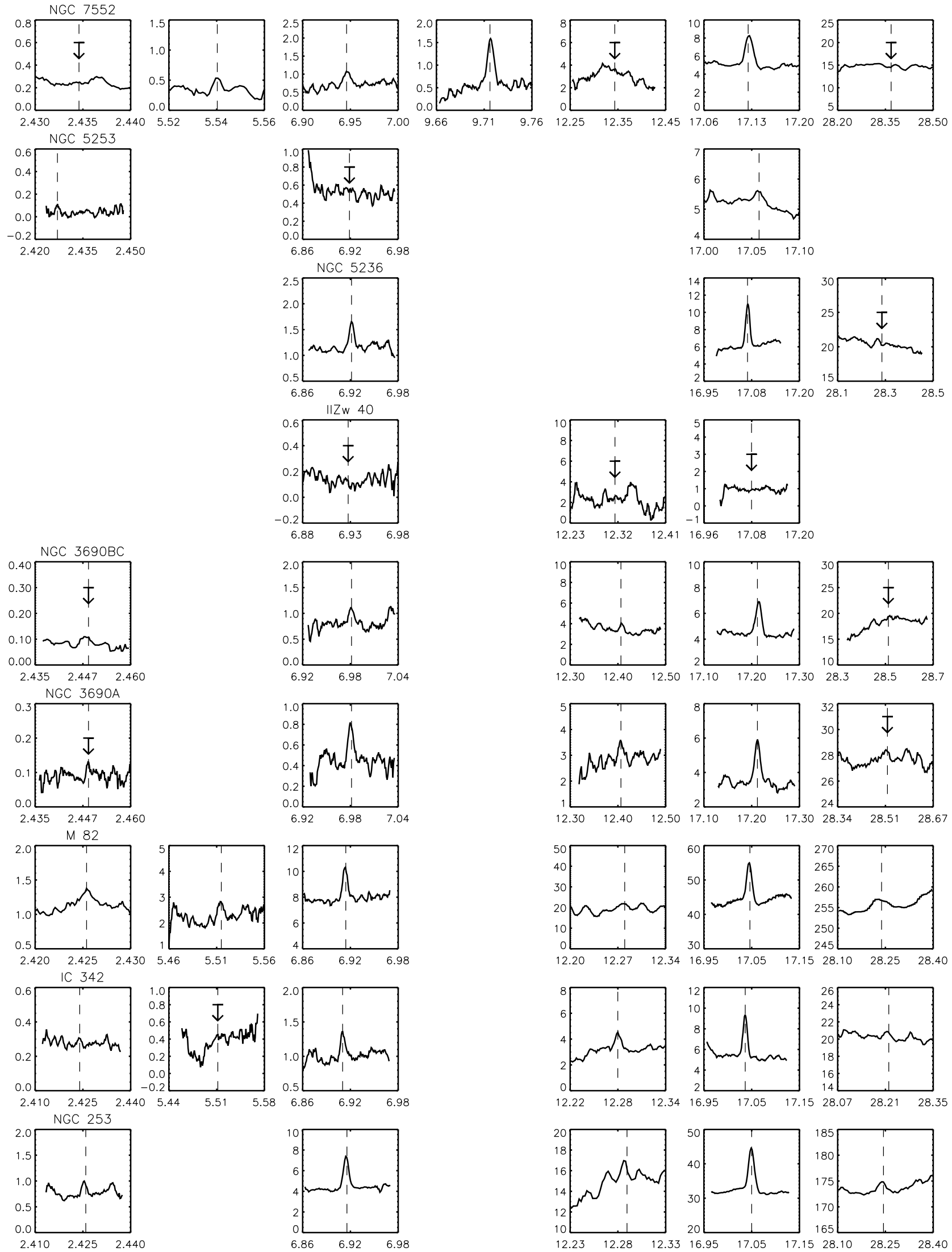

Fig. 1. $\mathrm{H}_{2}$ molecular lines from starbursts: from left to right $(1-0) \mathrm{Q}(3),(0-0) \mathrm{S}(7),(0-0) \mathrm{S}(5),(0-0) \mathrm{S}(3),(0-0) \mathrm{S}(2)$, (0-0) $\mathrm{S}(1)$ and $(0-0) \mathrm{S}(0)$. The vertical axis is Flux in $\mathrm{Jy}$, the horizontal axis is wavelength in $\mu \mathrm{m}$. Note: previously published spectra for NGC 3256, NGC 4038 and NGC 4945 and NGC 6946 are not included in this figure. 


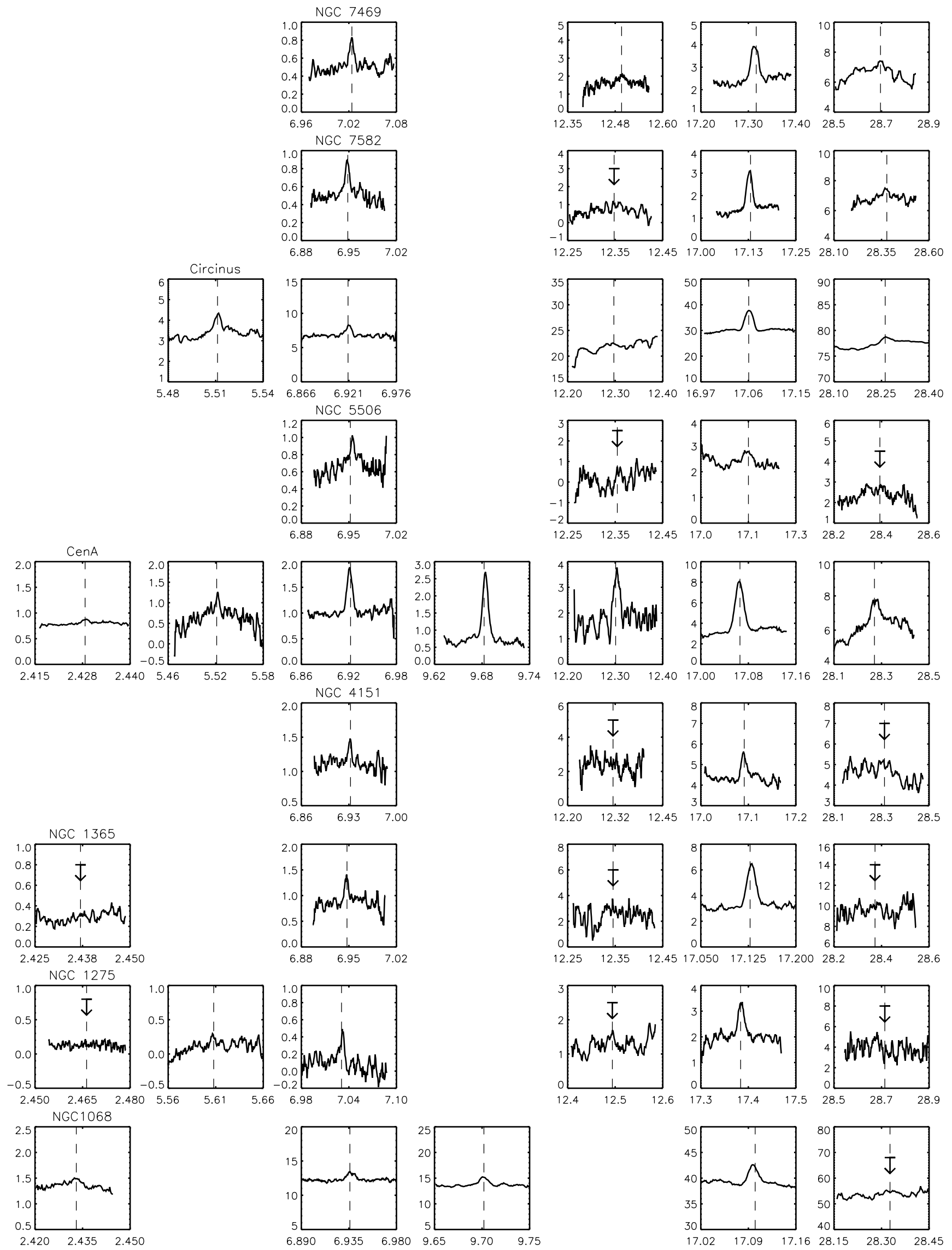

Fig. 2. $\mathrm{H}_{2}$ molecular lines in Seyferts. The spectral lines from the various transitions and the axes are the same as in Fig. 1. 
Table 1. The Starburst sample galaxies.

\begin{tabular}{|c|c|c|c|c|}
\hline Galaxy Name & $\begin{array}{c}D \\
\mathrm{Mpc}\end{array}$ & $\begin{array}{c}A_{V}^{1} \\
{[\mathrm{mag}]}\end{array}$ & Spec. type & comments \\
\hline NGC 253 & 3.5 & 30 & HII, SB & Sc, edge-on \\
\hline IC 342 & 3.6 & 12 & HII, SB & Scd, face-on \\
\hline II $\mathrm{Zw} 40$ & 7 & $3^{2}$ & HII & $\mathrm{BCD}$ \\
\hline $\mathrm{M} 82^{3}$ & 3.3 & 10 & HII, SB & Irr \\
\hline NGC $3256^{a}$ & 36.6 & 35 & SB & colliding pair \\
\hline NGC $3690 \mathrm{~A} / \mathrm{BC}^{3}$ & 40 & 20 & SB & pec. merger \\
\hline NGC $4038 / 39^{3, b}$ & 21.0 & 80 & SB & interacting \\
\hline NGC $4945^{c}$ & 4 & 20 & SB, Sy2 & Sc edge-on \\
\hline NGC 5236 (M 83) & 5.0 & 5 & HII, SB & $\mathrm{SAB}(\mathrm{s})$, face-on \\
\hline NGC 5253 & 4.1 & 14 & HII, SB & Im pec \\
\hline NGC $6946^{d}$ & 5.7 & $1.8^{4}$ & HII & $\mathrm{S}(\mathrm{AB}) \mathrm{cd}$,face-on \\
\hline NGC 7552 & 21.2 & 5 & HII, LINER & $\mathrm{SA}(\mathrm{c})$, face-on \\
\hline
\end{tabular}

${ }^{1} A_{V}$ from Genzel et al. (1998).

${ }^{2} A_{V}$ (screen) from Baldwin et al. (1982).

3 The ISO pointings on these galaxies are as follows (J2000): M 82:00 39 43.38, 6940 44.4, NGC 3690A: $114554.24,583346.5$, NGC 3690BC: $114554.06,5833$ 45.6, NGC 4038/39:00 48 07.66, -18 53 04.1. The ISO pointings for the remaining galaxies are on the galaxy's nucleus.

${ }^{4} A_{V}$ (screen) from Hyman et al. (2000).

${ }^{a}$ Rigopoulou et al. (1996), ${ }^{b}$ Kunze et al. (1996), ${ }^{c}$ Spoon et al. (2000), ${ }^{d}$ Valentijn et al. (1996).

Table 2. The Seyfert sample galaxies.

\begin{tabular}{ccccc}
\hline \hline Galaxy Name & $\begin{array}{c}D \\
\text { Mpc }\end{array}$ & $\begin{array}{c}A_{V} \\
{[\mathrm{mag}]}\end{array}$ & Spec. type & comments \\
\hline NGC 1068 & 14 & $8^{1}$ & Sy2 & SAb \\
NGC 1275 & 70 & $2^{2}$ & Sy2 & cD, pec. \\
NGC 1365 & 22.3 & $2.5^{2}$ & Sy1.8 & SBb \\
NGC 4151 & 20 & $3^{1}$ & Sy1.5 & SABab \\
Cen A & 4 & $30^{3}$ & Radio & SO pec. \\
NGC 5506 & 23 & $8^{3}$ & Sy1.9 & Sa pec. \\
Circinus & 3 & $20^{3}$ & Sy2 & SAb \\
NGC 7582 & 20 & $18^{3}$ & Sy2 & SBab \\
NGC 7469 & 66 & $20^{1}$ & Sy1.2 & SABa \\
\hline
\end{tabular}

${ }^{1} A_{V}$ mixed case (from Genzel et al. 1998).

${ }^{2}$ NGC 1275: Krabbe et al. (2000); NGC 1365: Kristen et al. (1997).

${ }^{3} \mathrm{~A}_{V}$ screen case (from Genzel et al. 1998).

where $\operatorname{flux}(i)$ is the flux of a line in the $i$ th state, $A(i)$ is the $A$-coefficient of that transition, $\nu(i)$ is the frequency of the transition, $h$ is Planck's constant, and beam is the solid angle of the beam size. In Figs. 3 and 4 we plot the natural logarithm of Eq. (1).

For the excitation diagrams we used extinctioncorrected line fluxes. We estimated the extinction for each line flux using the $A_{V}$ values listed in Tables 1 and 2. For $\lambda<8 \mu \mathrm{m}$ we used $A(\lambda) \propto \lambda^{(-1.75)}$ and for $\lambda>8 \mu \mathrm{m}$ the extinction law of Draine \& Lee (1984) and Draine (1989) were used. We assumed similar obscuration of the ionized and molecular media.

The excitation temperature of the line-emitting gas is the reciprocal of the slope of the excitation diagram, corresponding to the kinetic temperature in local thermodynamic equilibrium (LTE). Assuming thermal emission, it is apparent that $\mathrm{H}_{2}$ is present in a range of temperatures in all cases ${ }^{3}$. The excitation temperature changes rapidly with energy level. This is of course a natural consequence of the fact that the gas is in reality consisting of various components at various temperatures. The detections of the $\mathrm{S}(1)$ and $\mathrm{S}(0)$ lines (or the upper limits of the latter whenever not detected) are used to constrain the temperature of the "warm" line emitting gas, while the $\mathrm{S}(5)$ and $\mathrm{S}(7)$ lines are used to constrain the temperature of the somewhat "hotter" gas. In fact the $\mathrm{S}(5)$ and $\mathrm{S}(7)$ lines most likely probe the same excited gas as the near-infrared ro-vibrational lines. Therefore, the detection of the $\mathrm{S}(0)$ and $\mathrm{S}(1)$ lines is more important since we can probe the more abundant "warm" gas. In Tables 5 and 6 (Cols. 2 and 5 respectively) we list the temperatures (for the "warm" and the "hotter" gas) for the starbursts and Seyferts, respectively.

We note that the derived excitation temperature is affected by the total ortho-to-para conversion rate of $\mathrm{H}_{2}$. For the calculations above we have assumed an ortho-to-para abundance ratio of 3 , the equilibrium value for temperatures $T \geq 200 \mathrm{~K}$ (assuming LTE conditions). Sternberg \& Neufeld (1999) have measured an ortho-to-para ratio of 3 in the PDR star-forming region S140. Assuming a lower ortho-to-para conversion rate of 1 we find higher values

\footnotetext{
${ }^{3}$ We note that the assumption of LTE conditions implies densities higher than about $n>10^{4} \mathrm{~cm}^{-3}$ which may not be present if a diffuse component contributes significantly. In that case the excitation diagram can still be used as an empirical tool although the interpretation of excitation temperatures is not straightforward.
} 

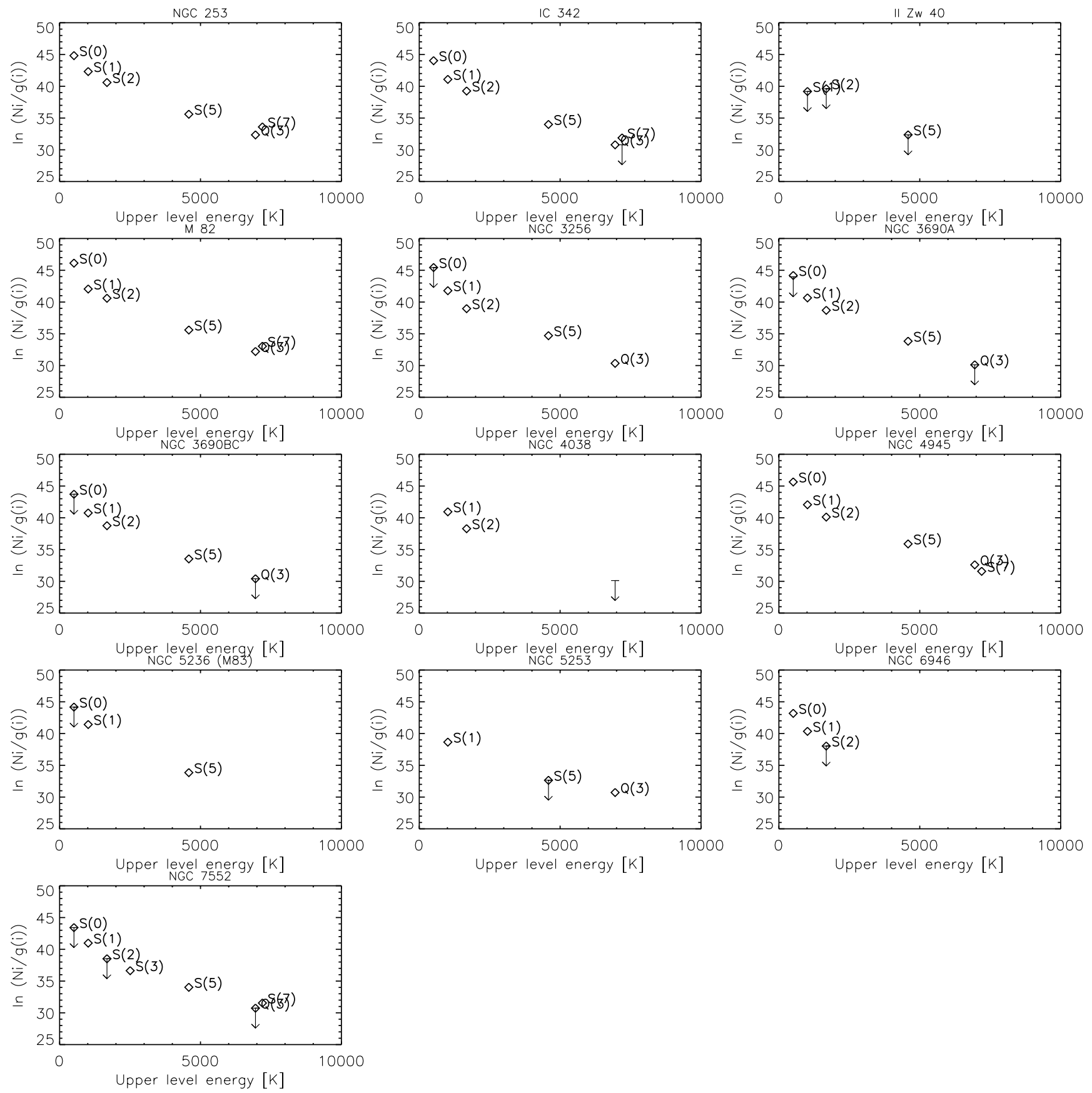

Fig. 3. Excitation diagrams for the starbursts.

of $T_{\mathrm{S}(0)-\mathrm{S}(1)}$ and lower values of $T_{\mathrm{S}(2)-\mathrm{S}(1)}$, internally inconsistent and implying that multiple temperature components should be considered to fit the data. We believe that under the assumption of LTE conditions an ortho-topara abundance ratio of 3 is a realistic value.

From the $\mathrm{S}(0)-\mathrm{S}(1)$ detections in starbursts we derive an average temperature of $T_{\mathrm{SB}} \sim 160 \pm 10 \mathrm{~K}$. For those starbursts for which there was no $\mathrm{S}(0)$ detection we used the $\mathrm{S}(1)$ and $\mathrm{S}(2)$ detections to find the range of temperatures. For the starbursts we find $150 \leq T_{\mathrm{SB}} \leq 330 \mathrm{~K}$. For the Seyferts, the $\mathrm{S}(0)$ line is detected in CenA, Circinus,
NGC 7582 and NGC 7469. Based on these detections we derive an average temperature $T_{\text {Sey }} \sim 150 \pm 12 \mathrm{~K}$. For those Seyferts with $\mathrm{S}(0)$ non-detections we derive limits in the range $120 \leq T_{\text {Sey }} \leq 370 \mathrm{~K}$ (using $\mathrm{S}(1)$ and $\mathrm{S}(2)$ ). Although the number of $\mathrm{S}(0)$ detections in the Seyfert sample is slightly smaller (in the present sample the $\mathrm{S}(0)$ line is detected in $5 / 10$ of the starbursts and $4 / 9$ of the Seyferts) it is obvious that the temperatures of the "warm" gas are, within the errors, similar in starbursts and Seyferts. 

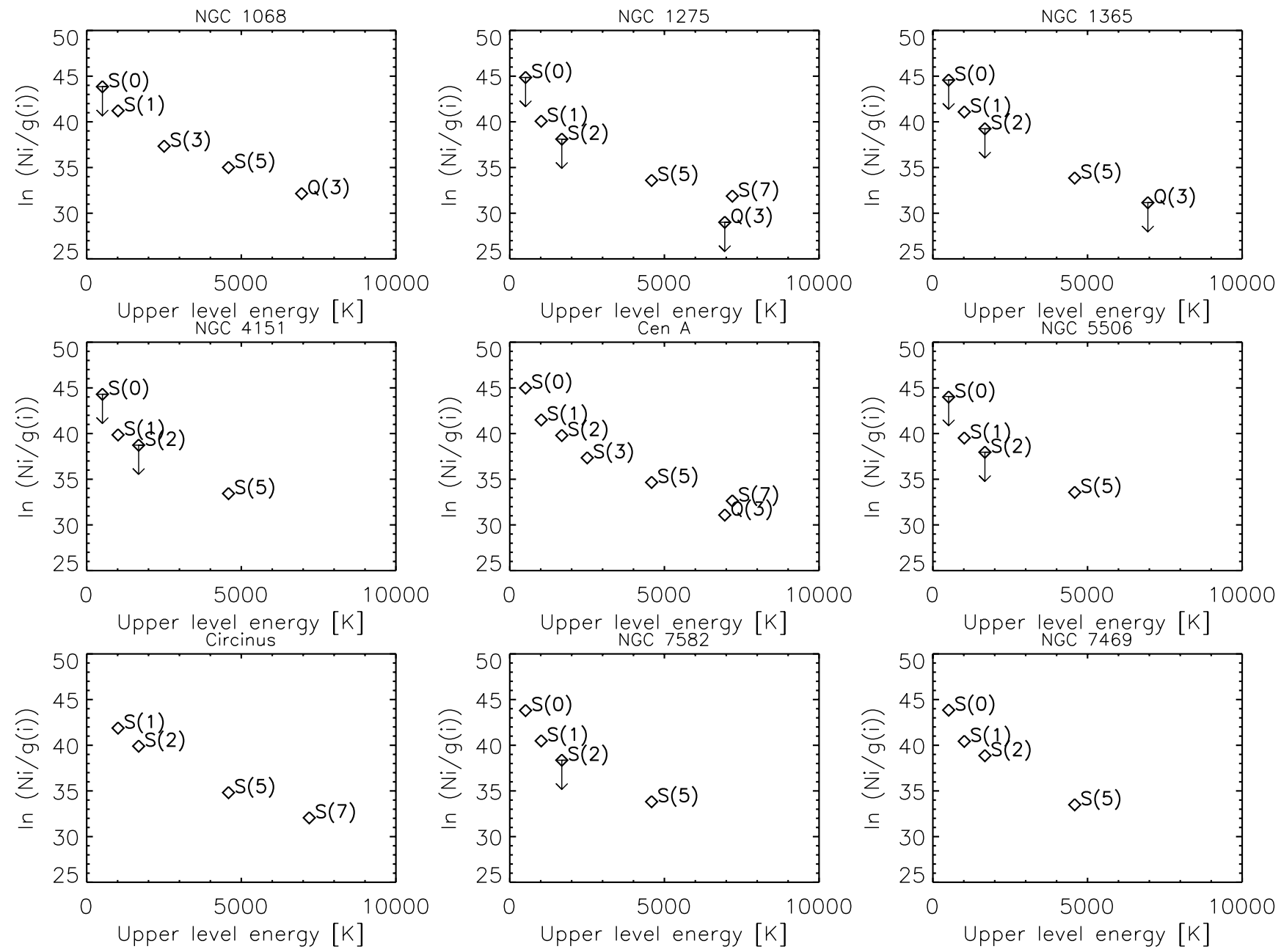

Fig. 4. Excitation diagrams for the Seyferts.

Table 3. $\mathrm{H}_{2}$ emission line fluxes - starbursts.

\begin{tabular}{cccccccc}
\hline \hline Galaxy Name & $(1-0) \mathrm{Q}(3)$ & $\mathrm{S}(7)$ & $\mathrm{S}(5)$ & $\mathrm{S}(3)$ & $\mathrm{S}(2)$ & $\mathrm{S}(1)$ & $\mathrm{S}(0)$ \\
$\lambda(\mu \mathrm{m})$ & 2.42 & 5.51 & $\begin{array}{c}6.91 \\
\times 10^{-20} \mathrm{~W} \mathrm{~cm}^{-2}\end{array}$ & 9.67 & 12.27 & 17.03 & 28.22 \\
& & & & & & \\
\hline NGC 253 & 2.80 & 8.40 & 11.5 & - & 12.0 & 19.57 & 2.13 \\
IC 342 & 0.39 & $<0.9$ & 1.60 & - & 2.50 & 4.90 & 0.80 \\
IIZw40 & - & - & $<0.45$ & - & $<4.5$ & $<0.85$ & - \\
M 82 & 2.65 & 4.80 & 11.50 & - & 12.0 & 15.0 & 7.8 \\
NGC 3256 & 0.38 & - & 4.70 & - & 2.35 & 11.50 & $<3.90$ \\
NGC 3690A & $<0.3$ & - & 1.97 & - & 1.8 & 3.7 & $<1.21$ \\
NGC 3690B/C & $<0.4$ & - & 1.46 & - & 1.92 & 4.11 & $<0.7$ \\
NGC 4038 & - & - & - & - & 1.6 & 3.95 & - \\
NGC 4945 & 3.2 & 1.11 & 15.4 & & 7.45 & 15.1 & 4.82 \\
NGC 5236 (M83) & - & - & 2.84 & - & - & 7.29 & $<1.04$ \\
NGC 5253 & 0.55 & - & $<0.60$ & - & - & 0.50 & - \\
NGC 6946 & - & - & - & - & $<0.93$ & 2.73 & 0.41 \\
NGC 7552 & $<0.86$ & 1.08 & 2.37 & 2.84 & $<1.5$ & 5.11 & $<0.52$ \\
\hline
\end{tabular}

$\mathrm{H}_{2}$ line fluxes from:

${ }^{a}$ Rigopoulou et al. (1996).

${ }^{b}$ Kunze et al. (1996).

${ }^{c}$ Spoon et al. (2000).

${ }^{d}$ Valentijn et al. (1996). 
Table 4. $\mathrm{H}_{2}$ emission line fluxes - Seyferts.

\begin{tabular}{|c|c|c|c|c|c|c|c|}
\hline Galaxy Name & $\overline{(11-0) \mathrm{Q}(3)}$ & $\overline{\mathrm{S}(7)}$ & $\overline{\mathrm{S}(5)}$ & $\overline{\mathrm{S}(3)}$ & $\overline{\mathrm{S}(2)}$ & $\mathrm{S}(1)$ & 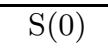 \\
\hline$\lambda(\mu \mathrm{m})$ & 2.42 & 5.51 & 6.91 & 9.67 & 12.27 & 17.03 & 28.22 \\
\hline \multicolumn{8}{|c|}{$\times 10^{-20} \mathrm{~W} \mathrm{~cm}^{-2}$} \\
\hline NGC $1068^{a}$ & 2.296 & - & 6.403 & 5.757 & - & 6.502 & $<1.872$ \\
\hline NGC 1275 & $<0.8$ & 0.9 & 1.55 & - & $<1.0$ & 2.06 & $<2.2$ \\
\hline NGC 1365 & $<0.85$ & - & 2.01 & - & $<3.13$ & 5.69 & $<1.65$ \\
\hline NGC 4151 & - & - & 1.32 & - & $<1.86$ & 1.667 & $<1.26$ \\
\hline CenA & 0.792 & 3.259 & 4.535 & 5.806 & 5.397 & 8.635 & 2.511 \\
\hline NGC 5506 & - & - & 1.508 & - & $<0.859$ & 1.185 & $<0.928$ \\
\hline Circinus & - & 2.72 & 7.97 & - & 2.36 & 13.94 & 1.56 \\
\hline NGC 7582 & - & - & 1.945 & - & $<1.289$ & 3.116 & 0.764 \\
\hline NGC 7469 & - & - & 1.394 & - & 2.125 & 2.955 & 0.8 \\
\hline
\end{tabular}

$\mathrm{H}_{2}$ line fluxes from: ${ }^{a}$ Lutz et al. (2000).

Taken at face value the similar temperatures found for starbursts and Seyferts indicate that the conditions in the gas are the same in both environments. Although this could have been the anticipated result (since we are looking at the same transitions originating in "warm" clouds in galaxies) we caution that a number of factors may contribute to this effect: first, most of the Seyferts in our sample are "mixed" objects that is, there is evidence for the presence of an extended starburst component (e.g. NGC 7469, NGC 7582, CenA and Circinus) whose energy output is comparable to that of the central active nucleus. Second, the large SWS apertures sample a mixture of molecular hydrogen emission from cool clouds in the extended circumnuclear regions as well as the warmer central clouds. Thus, effects of dilution of a pure AGNrelated effect by the more extended starburst activity are possible. Such a behaviour has already been noted in the case of NGC 1068 (Lutz et al. 2000) where the SWS- $\mathrm{H}_{2}$ emission traces gas originating both in the central active nucleus as well as the molecular ring which is present at larger spatial scales.

We next derive masses corresponding to the gas found at various temperatures, mostly the "warm" gas emitting the $\mathrm{S}(0)$ line as well as the "hotter" higher excitation gas in which the $S(7)$ line originates. The various masses are derived based on the following method: the column density (from Eq. (1)) is multiplied by the physical area of the object corresponding to the aperture. For aperture sizes we use the values quoted in Sect. 2 depending on the transition (either $\mathrm{S}(0)$ or $\mathrm{S}(7)$ ). The "warm" molecular masses were derived using the $\mathrm{S}(0)$ line (column density and temperature). In the cases of undetected $S(0)$ lines we have used the $\mathrm{S}(1)$ line (column density) and the $\mathrm{S}(1)$ $\mathrm{S}(2)$ temperature to derive the masses. The "hotter" gas masses are derived using the $\mathrm{S}(7)$ detections. The "warm" gas masses (denoted as $M_{1}$ ) and the "hotter" gas masses (denoted as $M_{2}$ ) are listed in Tables 5 and 6 (Cols. 3 and 6) for starbursts and AGN, respectively.

\section{Comparison with $\mathrm{CO}$ data}

Most of the knowledge about the molecular content of galaxies has so far relied on observations of $\mathrm{CO}$ emission, assuming a $\mathrm{CO} / \mathrm{H}_{2}$ conversion factor based on Galactic Giant Molecular Clouds (GMCs). However, CO probes successfully gas lying at low temperatures of a few tens up to a hundred. And although the low temperature gas comprises the bulk of the gas content in a galaxy, it provides no information on the "warmer" gas which might be more directly linked to the source of activity. In Sect. 3.2 we showed how ISO can give a firm estimate of the "warm" gas content of these galaxies.

Here, we try to estimate what fraction of the total gas mass lies in higher temperatures as we directly compare our new ISO findings with published $\mathrm{CO}$ data. Although for such a comparison higher $\mathrm{CO}$ transitions $(J=3 \rightarrow$ $2, J=6 \rightarrow 5$ etc. $)$ probing "warmer" gas $(T>50 \mathrm{~K})$ are more directly related to the gas seen by ISO however, these transitions are not available for the majority of the galaxies presented here. Such a detailed analysis has been carried out for only two galaxies, NGC 6946 (Valentijn et al. 1996), and NGC 4945 (Spoon et al. 2000).

In Tables 5 and 6 we compare the masses derived from the SWS $\mathrm{H}_{2}$ emission lines with mass estimates from $\mathrm{CO}$ observations (assuming the $\mathrm{GMC} \mathrm{CO} / \mathrm{H}_{2}$ conversion factor). The comparison is more meaningful for galaxies where the $\mathrm{S}(0)$ is detected because only then do we have an accurate estimate of the "warm" gas mass.

In the case of starbursts the fraction of "warm" gas ranges between 1 to $10 \%$ of the total gas mass. In Seyferts the fraction of the "warm" gas is overall higher. Although there is great variation among the different objects, in general, the "warm" gas makes up between 2 and $35 \%$ of the total gas (as probed by CO), a value deduced by using only those Seyferts with $\mathrm{S}(0)$ detections. For the mass of the "hotter" gas we find that it accounts for a lot less, of the order of at most $1 \%$ in both starbursts and Seyferts.

Although the gas in starbursts and Seyferts has similar temperatures the corresponding masses of "warm" gas are distinctively different. The amount of "warm" gas in Seyferts is one to two orders of magnitude higher than in starbursts. The origin of this difference could lie in the excitation mechanism. As we discuss in Sect. 6 apart from shocks and PDRs $\mathrm{H}_{2}$ may also arise in gas heated by hard X-ray photons originating in the central AGN. 
These X-ray photons are capable of penetrating large columns of gas thus heating extensive parts of the circumnuclear regions reaching out to the larger scale disks in AGN. Spatially resolved observations of rotational lines in AGN would be crucial in investigating the extent of the $\mathrm{H}_{2}$ emitting regions.

\section{5. $\mathrm{H}_{2}$ excitation mechanisms}

The $\mathrm{H}_{2}$ molecule can be excited via three distinct mechanisms:

(a) UV fluorescence where photons with $\lambda \geq 912 \AA$ are initially absorbed by the $\mathrm{H}_{2}$ molecule (in its Lyman \& Werner bands) and later re-emitted resulting in the population of the various vibration-rotation levels of the ground state (e.g. Black \& van Dishoeck 1987).

(b) Shocks where high-velocity gas motions in a quiescent cloud can heat, chemically alter and accelerate the ambient gas resulting in excitation of the $\mathrm{H}_{2}$ molecule (e.g. Hollenbach \& McKee 1989) and,

(c) X-ray illumination where hard X-ray photons are capable of penetrating deeply into molecular clouds and heating large amounts of gas (e.g. Maloney 1996).

To distinguish among the three primary excitation mechanisms is far from trivial: high UV radiation flux as well as high-velocity gas motions are both present in the nuclei of Seyfert galaxies and (to a lesser extent) of starbursts. UV radiation can originate either from the existing stellar population, from current star-forming processes, or the accretion disk. On the other hand, shock excitation can originate from the central engine, an outflow due to the large number of supernovae remnants or finally gas motions due to galaxy-galaxy interactions. In addition, the low rotational transitions arise from collisionally excited warm gas which complicates the distinction even further.

Since the signatures of all three mechanisms on the pure rotational lines are almost indistinguishable, an analysis including both ro-vribational and rotational lines would be ideal to address the issue of the excitation mechanism in extragalactic environments. However, the different apertures used to measure lines at different parts of the spectrum introduce large uncertainties, making such a study almost impossible. In what follows we present a brief account of the various theoretical model predictions and compare them to our observed line ratios. In addition, we compare our measurements for the two extragalactic samples to well-studied Galactic templates with the aim of better understanding the origin of the $\mathrm{H}_{2}$ emission.

\subsection{Comparison with theoretical work}

\subsubsection{PDR models}

In this section we compare the observed line ratios to the published PDR models of Burton Hollenbach \& Tielens (1992, BHT). From the present samples we find mean line ratios of $\mathrm{S}(1) / \mathrm{S}(0)=5.31 \pm 0.64$ and $3.75 \pm 1.38$ and $\mathrm{S}(1) / \mathrm{S}(2)=2.33 \pm 0.82$ and $1.85 \pm 0.93$ for starbursts and Seyferts, respectively. We find that the line ratios for the starbursts can be explained with a sensible combination of gas densities and radiation field strengths. For instance the mean $\mathrm{S}(1) / \mathrm{S}(0)$ starburst value can be reproduced by models 4 or 5 (from BHT) whereas to explain the $\mathrm{S}(1) / \mathrm{S}(2)$ ratio a harder radiation field is necessary (such as in model 7). The mean $\mathrm{S}(1) / \mathrm{S}(0)$ line ratio for Seyferts is somewhat but not significantly lower than the one in starbursts. Model 3 reproduces fairly closely the $\mathrm{S}(1) / \mathrm{S}(0)$ ratio in Seyferts but fail to predict the $\mathrm{S}(1) / \mathrm{S}(2)$ ratio which would again require a harder radiation field.

Although it is impossible to constrain PDR models based on the current observations such direct comparisons of the model prediction and the current measurements suggest that a combination of normal PDRs can explain the emission in both Starburst and Seyfert galaxies.

\subsubsection{Shock models}

A direct comparison of the various model predictions (e.g. Brand et al. 1989; Hollenbach \& McKee 1989; Kaufman \& Neufeld 1996) with our observed line ratios for starbursts and Seyferts shows that, a single speed shock model cannot explain the observed line ratios. Additionally, almost all models predict $S(1) / S(0)$ line ratios much larger than the observed ones (ie the predicted $0-0 \mathrm{~S}(0)$ emission is weaker). If the gas temperature is high then the $J=2$ level is not populated resulting in a weak $0-0 \mathrm{~S}(0)$ emission. In galactic sources with shocked $\mathrm{H}_{2}$ emission, the temperature of the higher levels (say $J=8$ to $J=3$ ) is relatively constant at about $700 \mathrm{~K}$ (Wright, priv. comm.) thus, the $J=2$ level does not emit strongly. However, in extragalactic systems the situation is probably different. The low velocity shocks cannot heat the gas to high enough temperatures, the $J=2$ level is populated resulting in enhanced $0-0 \mathrm{~S}(0)$ line emission compared to the various model predictions.

Among the Seyferts, NGC 1275 is a candidate for shock induced $\mathrm{H}_{2}$ emission. This is also supported by the rovibrational lines observed in the near-IR (Fischer et al. 1987; Krabbe et al. 2000). For the Starburst galaxies it is harder to make any predictions: except perhaps from the case of the luminous IR galaxy NGC 6240 (Egami et al. 2002 , in preparation), no other starburst galaxy is a clear case candidate for shock excited $\mathrm{H}_{2}$ emission.

\subsubsection{X-ray irradiated molecular gas}

So far, the efforts to model X-ray Dominated Regions (XDRs) have been concentrated mostly on X-rays originating from a central engine. The model predictions account mostly for the ro-vibrational line ratios and/or consider additional diagnostics such as the near-infrared [Fe II] $1.26 \mu \mathrm{m}$ and the cooling lines [OI] $63 \mu \mathrm{m}$ and [C II] $158 \mu \mathrm{m}$ (Maloney et al. 1996). Tiné et al. (1997) presented model predictions for the strength of the $\mathrm{S}(7)$ line, the only rotational transition so far included in XDR 
Table 5. Comparison of masses - starbursts.

\begin{tabular}{|c|c|c|c|c|c|c|c|}
\hline Galaxy Name & $\begin{array}{c}T(\mathrm{~S}(0)-\mathrm{S}(1)) \\
\mathrm{K}\end{array}$ & $\begin{array}{c}M_{1} \\
\times 10^{8} M_{\odot}\end{array}$ & $\begin{array}{c}T(\mathrm{~S}(1)-\mathrm{S}(2)) \\
\mathrm{K}\end{array}$ & $\begin{array}{c}T(\mathrm{~S}(5)-\mathrm{S}(7)) \\
\mathrm{K}\end{array}$ & $\begin{array}{c}M_{2} \\
\times 10^{3} M_{\odot}\end{array}$ & $\begin{array}{c}M_{\mathrm{CO}} \\
\times 10^{8} M_{\odot}\end{array}$ & $\overline{\text { Ref }}$ \\
\hline NGC 253 & 195 & 0.05 & 380 & 1275 & 6.0 & 5 & $(1)$ \\
\hline IC 342 & 171 & 0.05 & 365 & $<1075$ & 1.0 & 2 & (1) \\
\hline IIZw40 & - & - & - & - & - & - & \\
\hline M 82 & 120 & 0.54 & 450 & 1020 & 4.0 & 6 & (1) \\
\hline NGC 3256 & $\geq 135$ & 10 & 230 & - & - & 300 & (1) \\
\hline NGC 3690A & $\geq 140$ & - & 335 & - & - & 130 & $(2)^{a}$ \\
\hline NGC 3690B/C & $\geq 170$ & - & - & - & - & - & \\
\hline NGC 4038 & - & - & - & - & - & - & - \\
\hline NGC 4945 & 139 & - & - & - & - & 2.7 & (3) \\
\hline NGC 5236 (M83) & $\geq 182$ & 2.0 & - & - & - & - & - \\
\hline NGC 5253 & - & - & - & - & - & - & \\
\hline NGC 6946 & 174 & 0.03 & $\leq 290$ & - & - & 0.3 & $(4)$ \\
\hline NGC 7552 & $\geq 158$ & 0.8 & $\leq 272$ & 1120 & 30 & 80 & (1) \\
\hline
\end{tabular}

References: (1) Aalto et al. (1995); (2) Young et al. (1986); (3) Henkel et al. (1994); (4) Sage et al. (1990).

${ }^{a} \mathrm{CO}$ value refers to NGC $3690 \mathrm{~A} / \mathrm{B}$.

Table 6. Comparison of masses - Seyferts.

\begin{tabular}{cccccccc}
\hline \hline Galaxy Name & $T(\mathrm{~S}(0)-\mathrm{S}(1))$ & $M_{1}$ & $T(\mathrm{~S}(1)-\mathrm{S}(2))$ & $T(\mathrm{~S}(5)-\mathrm{S}(7))$ & $M_{2}$ & $M_{\mathrm{CO}}$ & Ref \\
& $\mathrm{K}$ & $\times 10^{8} M_{\odot}$ & $\mathrm{K}$ & $\mathrm{K}$ & $\times 10^{3} M_{\odot}$ & $\times 10^{8} M_{\odot}$ & Ref \\
\hline NGC 1068 & $\geq 145$ & 1.0 & - & - & - & 40 & $(1)$ \\
NGC 1275 & $\geq 115$ & 25 & 340 & 1170 & 3.0 & 60 & $(2)$ \\
NGC 1365 & $\geq 145.0$ & 1.7 & 365 & - & - & - & \\
NGC 4151 & $\geq 115$ & 0.1 & $\leq 590$ & - & - & 0.3 & $(3)$ \\
CenA & 145 & 0.11 & 390 & 1300 & 2.0 & 1.0 & $(4)$ \\
NGC 5506 & $\geq 112$ & 2.0 & $\leq 430$ & - & - & 3 & $(3)$ \\
Circinus & 199 & 0.8 & 221 & 555 & 1.53 & 30 & $(5)$ \\
NGC 7582 & 153 & 0.76 & $\leq 315$ & - & - & 100 & $(6)$ \\
NGC 7469 & 145 & 54 & 425 & - & - & 150 & $(3)$ \\
\hline
\end{tabular}

References: (1) Sage et al. (1990); (2) Bridges \& Irwin (1998); (3) Rigopoulou et al. (1997); (4) Wild et al. (1997); (5): Curran et al. (1998); (6) Maiolino et al. (1997)

models. In accordance with the results of Maloney et al. (1996), Tiné et al. found that the $\mathrm{H}_{2}$ emission arises in typical temperatures $T<1000 \mathrm{~K}$ and relatively high densities. For higher temperatures collisional excitation dominates the low vibrational levels. We note that the $\mathrm{H}_{3}^{+}$lines characteristic of $\mathrm{X}$-ray irradiated gas (Draine \& Woods 1990) have not been detected in any of our sample starbursts or Seyferts, therefore it is difficult to make any predictions. An exception is NGC 1068 where upper limits in $\mathrm{H}_{3}^{+}$have been reported in Lutz et al. (2000) but, unfortunately, provide no constraints on the models.

From the comparisons of our measurements to theoretical models we conclude that much of the $\mathrm{H}_{2}$ emission in starbursts could originate in PDRs although a combination of PDRs and shocks of various speeds is also a viable solution. A similar situation is probably true for the Seyferts as well, although as we already discussed in Sect. 4 X-rays from the central AGN may also play an important role in heating up large amounts of gas and triggering $\mathrm{H}_{2}$ emission.

Through the exercise of comparing our observations with theoretical models we want to emphasize how difficult it is to discriminate between the various $\mathrm{H}_{2}$ emission mechanisms and that in order to investigate thoroughly the origin of the $\mathrm{H}_{2}$ emission in galaxies a host of vibrational and rotational transitions must be available.

\subsection{The empirical way: Comparison with Galactic templates}

In this section we compare our measurements to a number of well studied local Galactic templates. For such a comparison we have considered two typical PDR regions "S140" (Timmermann et al. 1996) and the Orion Bar (Rosenthal 2000, priv. comm), and two cases of shockpowered emission "Cepheus A" (Wright et al. 1996) and Orion Peak 1 (Rosenthal et al. 1999). In Table 9 we list the temperatures and masses of the local Galactic templates along with "mean" properties of our sample starburst and Seyfert galaxies. We note that for the mean values quoted in Table 9 we have used all sample galaxies.

The average temperatures for the starburst and Seyfert galaxies are the same as the temperature observed in both cases of PDR emission and well below what is observed for 
Table 7. Comparison with galactic templates.

\begin{tabular}{cccc}
\hline \hline Type & $\mathrm{S}(1) / \mathrm{S}(0)$ & $\mathrm{S}(1) / \mathrm{S}(2)$ & $T(\mathrm{~S}(0)-\mathrm{S}(1))$ \\
& & & $\mathrm{K}$ \\
\hline Starbursts & 5.3 & 2.33 & $160-(330)^{1}$ \\
Seyferts & 3.75 & 1.85 & $150-(370)^{1}$ \\
S140 (PDR) & - & 1.00 & 159 \\
Orion Bar (PDR) & 1.11 & 5.29 & 155 \\
Cepheus A West (shock) & & 0.54 & 700 \\
Orion Peak 1 (shock) & - & 0.96 & $(570)^{2}$ \\
\hline
\end{tabular}

1 The upper limit of $370 \mathrm{~K}$ is derived from $\mathrm{S}(1)$ and $\mathrm{S}(2)$ transitions for those Seyferts where the $\mathrm{S}(0)$ is not detected.

${ }^{2}$ Temperature value derived from $\mathrm{S}(1)$ and $\mathrm{S}(2)$ transitions.

an Orion type shock. Overall, although in reality different regions and excitation mechanisms contribute to the galaxy-integrated temperature distribution these simple comparisons suggest that normal PDRs are responsible for most of the emission in starburst and Seyfert galaxies.

\section{PAH and $\mathrm{H}_{2}$ emission}

It has been known for some time (see e.g. review by Puget \& Lèger 1989) that the $3-12 \mu \mathrm{m}$ part of the spectrum of galaxies is dominated by a number of emission features that appear whenever the interstellar medium is exposed to moderately intense UV radiation. The exact nature of the carriers that are responsible for the emission is most likely large carbon-rich molecules the so-called Polycyclic Aromatic Hydrocarbons (PAH). With ISO it has been possible to detect $\mathrm{PAH}$ emission in a number of galactic (e.g. Verstraete et al. 1996; Roelfsema et al. 1996) and extragalactic (e.g. Rigopoulou et al. 1999) environments. Here, we want to investigate whether any link exists between PAH emission (ultimately related to the presence of PDRs and HII regions) and rotational $\mathrm{H}_{2}$ emission originating in the molecular clouds of galaxies where stars are formed. If both warm $\mathrm{H}_{2}$ emission and $\mathrm{PAH}$ emission originate mostly in UV-illuminated surfaces of molecular clouds (i.e. PDRs), their luminosities should correlate well when averaged over a galaxy.

In Fig. 5 we plot the $7.7 \mu \mathrm{m}$ PAH luminosity $L_{7.7}$ vs. the $\mathrm{H}_{2} \mathrm{~S}(1)$ luminosity $L_{\mathrm{H}_{2} \mathrm{~S}(1)}$ for our sample starbursts and AGN. We find that starbursts are consistent with a relation of the type $\log L_{7.7}=C+0.69 \log L_{\mathrm{H}_{2} \mathrm{~S}(1)}$. Some Seyferts deviate strongly from the $\mathrm{H}_{2} / \mathrm{PAH}$ relation in having very weak $\mathrm{PAH}$ for their $\mathrm{H}_{2}$ luminosity. Seyferts as an entire sample (ie including those with $\mathrm{PAH}$ upper limits) follow the relation $\log L_{7.7}=C+0.48 \log L_{\mathrm{H}_{2} \mathrm{~S}(1)}$ which becomes $\log L_{7.7}=C+0.53 \log L_{\mathrm{H}_{2} \mathrm{~S}(1)}$ if we only use AGN with firm PAH detections. Finally, we have calculated the relation for both starbursts and AGN (with firm detections) and found it to be $\log L_{7.7}=C+$ $0.64 \log L_{\mathrm{H}_{2} \mathrm{~S}(1)}$, quite similar in slope to the one found for starbursts.

Overall we find that the $7.7 \mu \mathrm{m}$ and $\mathrm{H}_{2} \mathrm{~S}(1)$ luminosities of starbursts and AGN (with firm detections) are

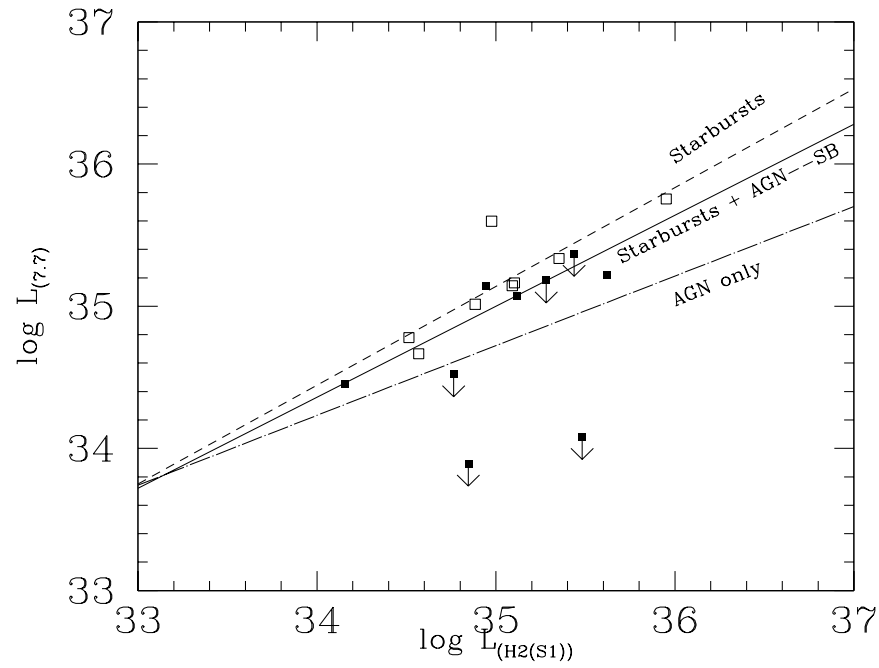

Fig. 5. PAH luminosity $L_{7.7}$ vs. the $\mathrm{H}_{2} \mathrm{~S}(1)$ luminosity for our sample starbursts and AGN. The open and filled squares represent the Starburst and AGN points, respectively. The lines shown represent the least square fits for: dashed line: starbursts, straight line: starbursts and AGN detections, longdashed line AGN only (non-detection included).

well correlated implying that the $\mathrm{PAH}$ and $\mathrm{H}_{2}$ emission have a common starburst origin. Indeed, the four AGN with firm PAH detections, CenA, NGC 7582, Circinus and NGC 7469, all have substantial starburst activity (e.g. Genzel et al. 1995 on NGC 7469, Maiolino et al. 1998 on Circinus etc.). Among the remaining five Seyferts three of them, NGC 4151, NGC 1275 and NGC 5506 are AGN dominated (with little or no Starburst contribution). These three AGN are significantly underluminous in PAH although they are relatively strong $\mathrm{H} 2$ emitters. A possible scenario to explain this behaviour is the following: in starbursts and in AGN with a significant large scale starburst component, $\mathrm{PAH}$ and $\mathrm{H}_{2}$ emission correlate well. In both cases the emission most likely originates in the starburst. In the remaining "pure" type $\mathrm{AGN}, \mathrm{H}_{2}$ emission is considerably stronger in comparison to the relatively weaker PAH emission. There are two possibilities for the high $\mathrm{H}_{2}$ / $\mathrm{PAH}$ ratio: either the $\mathrm{PAH}$ molecules are destroyed in the intense radiation field (possibly by the nuclear Xrays) or energetic photons escaping from the central X-ray source heat a large fraction of the circumnuclear material which in turns gives rise to the enhanced $\mathrm{H}_{2}$ emission (the $\mathrm{PAH}$ emission remains normal). Although it is very difficult to discriminate between these two options the current observational evidence tends to favor the latter possibility. As we already discussed in Sect. 4 the gas masses responsible for the $\mathrm{H}_{2}$ emission comprise a larger fraction of the total available gass mass (as traced by $\mathrm{CO}$ ) in AGN than in starbursts.

We conclude that the central AGN is probably responsible for the bi-modal behaviour of the $\mathrm{PAH}-\mathrm{H}_{2}$ relation in Starburst and Seyferts not by suppressing the PAH emission instead, by enhancing the $\mathrm{H}_{2}$ emission by heating larger fractions of the available circumnuclear gas. 


\section{Conclusions}

We have presented pure rotational $\mathrm{H}_{2}$ emission lines observed with the ISO satellite from a sample of starburst and Seyfert galaxies. We have compared the emission properties of the two samples. The results are summarized as follows:

The lowest $\mathrm{S}(0)$ transition has been detected in 5 out of the 10 starbursts and 4 out of the 9 Seyfert galaxies. The $\mathrm{S}(1) / \mathrm{S}(0)$ line ratio is within the errors not that different in starbursts and in Seyferts.

The temperature of the "warm" gas, as estimated using the $\mathrm{S}(0)$ detections was found to be similar (within errors) $T \sim 150 \mathrm{~K}$, in both starburst and Seyfert galaxies. This in turn implies that the global properties of the gas in both environments are the same. We caution though that due to the large ISO beams dilution of a pure AGN-related effect is important.

The "warm" gas mass constitutes up to $10 \%$ of the total molecular gas content (as traced by CO molecular observations) in starbursts. However, the fraction of "warm" gas in Seyferts is considerably higher reaching up to $35 \%$ (although with a large scatter in values). We propose that, extra "heating" of the gas is provided by energetic hard X-ray photons originating from the central AGN.

We have compared the observed strength of the molecular $\mathrm{H}_{2}$ lines with theoretical model predictions as well as those of local Galactic templates. Such comparisons reveal that a combination of various PDR clouds explain reasonably the line ratios observed in starbursts. Although such a combination of PDR models can also match the observed line ratios in Seyferts it is likely that slow velocity shocks and some heating from the central X-rays are also present.

Finally, we have examined the existence of a link between PAH and molecular line emission. We find that starbursts and Seyferts with a strong starburst-component follow a very similar correlation. On the other hand the ratio $\mathrm{H}_{2} / \mathrm{PAH}$ is higher in pure "AGN-dominated" objects. It is likely that an extended circumnuclear component of "warm" gas (heated by the nuclear X-ray emission) is present in AGN in which enhanced $\mathrm{H}_{2}$ emission originates. This "warm" gas component is either too far away for UV photons to reach or, acts as a shield to UV photons resulting in both cases in suppressed $\mathrm{PAH}$ emission.

Acknowledgements. We thank Amiel Sternberg, Alfred Krabbe and the anonymous referee for useful comments which significantly improved the present work. We thank Eckhard Sturm for help with the data reduction. SWS and the ISO Spectrometer Data Center at MPE are supported by DLR (DARA) under grants 50 QI 86108 and 50 QI 94023.

\section{References}

Aalto, S., Booth, R. S., Black, J. H., \& Johansson, L. E. B. 1995, A\&A, 300, 369

Alexander, T., Sturm, E., Lutz, D., et al. 1999, ApJ, 512, 204

Baldwin, J., Spinrad, H., \& Terlevich, R. 1982, MNRAS, 198, 535
Black, J. H., \& Dishoeck, E. F. 1987, ApJ, 322, 412

Bridges, T. J., \& Irwin, J. A. 1998, MNRAS, 300, 967

Burton, M. G., Hollenbach, D. J., \& Tielens, A. G. G. 1992, ApJ, 399, 563

Curran, S. J., Johansson, L. E. B., Rydbeck, G., \& Booth, R. S. 1998, A\&A, 338, 863

deGraauw, Th., Haser, L. N., Beintema, D. A., et al. 1996, A\&A, 315, L49

Draine, B. T. 1980, ApJ, 241, 1021

Draine, B. T. 1989, in ESA, Infrared Spectroscopy in Astronomy, 93

Draine, B. T., Roberge, W. G., \& Dalgarno, A. 1983, ApJ, 264, 485

Draine, B. T., \& Lee, H. M. 1984, ApJ, 285, 89

Draine, B. T., \& Woods, D. T. 1990, ApJ, 363, 464

Feuchtgrüber, H., Lutz, D., Beintema, D. A., et al. 1997, ApJ, 487, 962

Fischer, J., Smith, H. A., Geballe, T. R., Simon, M., \& Storey, J. W. V. 1987, ApJ, 320, 667

Genzel, R., Weitzel, L., Tacconi-Garman, L. E., et al. 1995, ApJ, 444, 129

Genzel, R., Lutz, D., Sturm, E., et al. 1998, ApJ, 498, 579

Habing, H. J. 1968, Bulletin of the Astronomical Institute of The Netherlands, 19, 421

Heckman, T. M., Butcher, H. R., Miley, G. K., \& van Breugel, W. J. M. 1981, ApJ, 247, 403

Henkel, C., Whiteoak, J. B., \& Mauersberger, R. 1994, A\&A, 284,17

Hollenbach, D., \& McKee, C. F. 1989, ApJ, 342, 306

Hyman, S. D., Lacey, C. K., Weiler, K. W., \& Van Dyk, S. D. 2000, AJ, 119, 1711

Joseph, R. D., Wade, R., \& Wright, G. S. 1984, Nature, 311, 132

Kaufman, M. J., \& Neufeld, D. A. 1996, ApJ, 456, 611

Kessler, M., Steinz, J. A., Anderegg, M. E., et al. 1996, A\&A, 315, L27

Krabbe, A., Sams, B. J., III, Genzel, R., et al. 2000, A\&A, 354, 439

Kristen, H., Jorsater, S., Lindblad, P., \& Boksenberg, A. 1997, A\&A, 328, 483

Kunze, D., Rigopoulou, D., Lutz, D., et al. 1996, A\&A, 315, L101

Lutz, D., Genzel, R., Sturm, E., et al. 1997, ISO Spectroscopy of Luminous Galaxies, in Proceedings of the first ISO workshop on Analytical Spectroscopy, ed. A. M. Heras, K. Leech, N. R. Trams, \& M. Perry, ESA Publications Division, 419, 143

Lutz, D., Genzel, R., Sturm, E., et al. 2000, ApJ, 536, 697

Maloney, P. R., Hollenbach, D. J., \& Tielens, A. G. G. M. 1996, ApJ, 466, 561

Maiolino, R., Ruiz, M., Rieke, G. H., \& Papadopoulos, P. 1997, ApJ, 485, 552

Maiolino, R., Krabbe, A., Thatte, N., \& Genzel, R. 1998, ApJ, 493, 650

Maloney, P. R. 1996, Ap\&SS, 248, 105

Moorwood, A. F. M., \& Oliva, E. 1988, A\&A, 203, 278

Moorwood, A. F. M., Lutz, D., Oliva, E., et al. 1996, A\&A, 315, L109

Oke, J. B., \& Sargent, W. L. W. 1968, AJ, 73, 895

Osterbrock, D. E. 1979, AJ, 84, 901

Puget, J.-L., \& Lèger, A. 1989, ARA\&A, 27, 161

Puxley, P. J., Hawarden, T. G., \& Mountain, C. M. 1988, MNRAS, 234, 29, 
Rigopoulou, D., Lutz, D., Genzel, R., et al. 1996, A\&A, 315, Timmermann, R., Bertoldi, F., Wright, C. M., et al. 1996, L125 A\&A, 315, 281

Rigopoulou, D., Papadakis, I., Lawrence, A., \& Ward, M. 1997, Tiné, S., Lepp, S., Gredel, R., \& Dalgarno, A. 1997, ApJ, 481, A\&A, 327, 493 282

Rigopoulou, D., Spoon, H. W. W., Genzel, R., et al. 1999, AJ, 118, 2625

Roelfsema, P. R., Cox, P., Tielens, A. G. G. M., et al. 1996, A\&A, 315, L289

Rosenthal, D., Bertoldi, F., \& Drapatz, S. 1999, A\&A, 356, 705

Sage, L. J., Shore, S. N., \& Solomon, P. M. 1990, ApJ, 351, 422

Sanders, D. B., Scoville, N. Z., \& Soifer, B. T. 1991, ApJ, 370, 158

Usuda, T., Sugai, H., Kawabata, H., et al. 1996, ApJ, 464, 818

Valentijn, E. A., van der Werf, P. P., de Graauw, T., \& deJong, T. 1996, A\&A, 315, 145

Valentijn, E. A., \& van der Werf, P. P. 1999, ApJ, 522, 29

Van der Werf, P. P., Genzel, R., Krabbe, A., et al. 1993, ApJ, 405,522

Verstraete, L., Puget, J.-L., Falgarone, E., et al. 1996, A\&A, 315, L337

Wild, W., Eckart, A., \& Wiklind, T. 1997, A\&A, 322, 419

Schaeidt, S. G., Morris, P. W., Salama, A., et al. 1996, A\&A, 315, L55

Wright, C. M., Drapatz, S., Timmermann, R., et al. 1996, A\&A, 315, L301

Spoon, H. W. W., Koornneed, J., Moorwood, A. F. M., et al. Young, J. S., Kenney, J. D., Tacconi, L. J., et al. 1986, ApJ, 2000, A\&A, 357, 898 311, L17

Sternberg, A., \& Neufeld, D. A. 1999, ApJ, 516, 371 